\title{
Pulse Intravenous Clomipramine as an alternative antidepressant treatment to ECT. A pilot study
}

\author{
Maj-Liz Persson* \\ Mats Adler* \\ Jerker Hetta** \\ * Department of Clinical Neuroscience, \\ Division of Psychiatry, Karolinska University \\ Hospital in Huddinge, Stockholm \\ ** Karolinska Institutet, Department of \\ Clinical Neuroscience, Section of \\ Psychiatry, Karolinska University Hospital in \\ Huddinge, Stockholm \\ SWEDEN
}

\begin{abstract}
Background and Objectives: The aim of the study was to examine the antidepressant effect of a single pulse dose of intravenous clomipramine (200 mg i.v.) followed by oral administration as an alternative method to electroconvulsive therapy.

Methods: Twenty-one inpatients (8 male, 13 female) with major depression were included. Depression severity was measured by Montgomery Asberg Rating Scale (MADRS) and Clinical Global Impression severity scale (CGI-S) before the pulse dose and 1 week after. The day after the pulse dose, the patient was medicated with $75 \mathrm{mg}$ of oral clomipramine and from day two with $150 \mathrm{mg}$ clomipramine daily.

Results: The MADRS score dropped with $39 \% \pm 22 \%$ and the CGI score with $28 \% \pm$ $19 \%$ in one week. The improvement of the MADRS score after one week was 13.1 (C.I.9.5-17.0). CGI-ratings dropped from a mean of 5.5 (SD 1.2) to 3.9 (SD 1.1), an improvement of $28 \% \pm 19 \%$.(C.I. 1.0-2.1). Both improvements were significant ( $\mathrm{p}<000.1)$.

Conclusions: Single pulse dose clomipramine administration ameliorates depressive symptoms, and may be an alternative to ECT.
\end{abstract}

Received 27 December 2006

Revised 29 June 2007

Accepted 24 July 2007 


\section{Background and Objectives}

Since the introduction of tricyclics and subsequently followed by the selective serotonin reuptake inhibitors (SSRIs) and lately SNRIs as antidepressants, delayed onset of action and lack of response have been significant clinical problems in the treatment of depression. The cause of delayed onset of therapeutic response is not known, but a hypothesis is that the pharmacokinetic characteristics of these medications may play a role $^{1}$. ECT is often a treatment of choice in treatment refractory depressions, but its role is limited by several factors as patient acceptance, high costs and, in some countries, legal restrictions. ECT given three times weekly takes between two and five weeks, often requiring in-patient care in a hospital. There is a need for alternative treatments, which preferably have a quick onset of action, high response rate and low costs.

Chiodo et al. $^{2}$, extrapolating from animal experiments, suggested that a single treatment with an antidepressant could be as effective as repetitive treatments. Pulse loading versus gradual dosing of intravenous clomipramine in outpatients with obsessive-compulsive disorder has been tested. A significantly greater improvement was achieved for the pulse loading group compared to the gradual dosing group by the end of week $1^{3}$. The main pharmacodynamical effect of clomipramine is to inhibit neuronal re-uptake of noradrenaline and serotonin in the synaptic cleft. Clomipramine undergoes first-pass metabolism with production of the active metabolite desmethylclomipramine, which is a selective noradrenergic re-uptake inhibitor. With gradually increasing dosing of clomipramine the plasma concentrations of desmethylclomipramine (noradrenergic action) is $40-85 \%$ higher than the concentration of clomipramine at a dose level of $75 \mathrm{mg} /$ day $^{4}$. By pulse dose administration the first pass metabolism is avoided and a high level of clomipramine, which is both serotonergic and noradrenergic active (dual action), is rapidly achieved, which could lead to a more rapid and robust antidepressant effect ${ }^{5}$. In 1989, Pollock et al. ${ }^{6}$ performed a double-blind, randomized trial of oral vs. intravenous clomipramine hydrochloride pulse-loading dosing regimens on 22 inpatients with a diagnosis of major depressive disorder (score on 21-item Hamilton Depression Rating scale, $\geq 18)^{7}$. Patients were given either an evening infusion of $150 \mathrm{mg}$ of clomipramine hydrochloride and placebo tablets or $150 \mathrm{mg}$ of oral clomipramine hydrochloride and an isotonic saline infusion. Twenty-four hours later, this procedure was repeated using a dose of $200 \mathrm{mg}$ clomipramine hydrochloride. No medication over the next five days was then given. The mean Hamilton Depression Rating Scale score for all patients, five days after pulse loading, had dropped by $35 \%$. This improvement was significant. There were no significant differences in either efficacy or side effects between the two groups.

Another double blind, placebo-controlled trial on sixteen nonsuicidal adolescents with major depressive disorder was designed by Sallee et ll $^{8}$. In this study, a single dose of clomipramine (200 mg i.v., $\mathrm{n}=8$ ) was compared with saline placebo $(n=8)$. The adolescents who received pulse clomipramine treatment demonstrated significant decreases in Hamilton Depression Rating Scale scores from baseline at 6 days but not at 36 hours. There was a significant difference from the effect of placebo. The intravenous pulse clomipramine was well tolerated. 


\section{Methods}

The subjects studied were inpatients at the psychiatric clinic, Karolinska University Hospital Huddinge, Sweden. Patients included in the study were diagnosed as suffering from major depressive disorder (MDD) and were waiting for ECT or were negative to that treatment. The MADRS depression rating scale consisting of 10 items, each rated 0 to 6 , was used ${ }^{9}$. Patients who scored above 20 on the MADRS were included in the study. A new MADRS-scoring was performed 1 week after the pulse dose. Ratings of the overall severity of the patient's condition were done at the same occasions (CGI) ${ }^{10}$.

Patients were examined with blood status including hematocrit, sodium, potassium, creatinine, glucose, blood pressure, pulse and electrocardiogram (ECG). Antidepressive medication was seponated one day before the pulse dose. A butterfly cannula was set 30 minutes before the clomipramine infusion was initiated so that vasovagal reactions could be differentiated from drug reactions. The patient then received $200 \mathrm{mg}$ clomipramine hydrochloride diluted in $500 \mathrm{ml}$ of normal saline during 3 hours. Blood pressure and pulse were measured every $30 \mathrm{~min}$ utes. The day after the pulse dose, the patients were medicated with oral clomipramine 75 mg per day and the dose was increased to 150 $\mathrm{mg}$ daily the day after. The data for this study was collected during routine clinical practice, and the patients were not randomized to treatments. The study constituted a part of the ongoing quality assurance program of our established treatments. Thus informed consent according to the Helsinki declaration is not required. The guideline of the research ethics review committee of the Karolinska Institutet stipulates that such studies, designed for quality assurance of routine treatments, are exempt from research ethics review.

\section{Statistical analyses}

Paired sample T-test of MADRS and CGI-scores before and six days after the clomipramine infusion was performed using the statistical program SPSS 13.0.

\section{Results}

Twenty-three inpatients entered the study, of which twenty-one completed the protocol. One patient was discontinued from the study because of extreme dizziness, and one with co-morbid personality disorder was not scored 1 week after the pulse dose. The mean initial MADRS score one day before the pulse loading was 34.4 (SD 7.6). One week after pulse loading, the mean MADRS score was 21.1 (SD 8.9), an improvement of $39 \% \pm$ $22 \%$. The improvement of the MADRS score was 13.1 (C.I. 9.5-17.0, p < 000.1) (Fig. 1). CGI-ratings dropped from a mean of 5.5 (SD 1.2) to 3.9 (SD 1.1), an improvement of $28 \%$ $\pm 19 \%$. Also this improvement of 1.6 was significant (C.I. 1.0-2.1, p < 000.1) (Fig. 2). Drowsiness and nausea were common during the pulse dosing treatment, but no serious adverse reactions occurred.

\section{Conclusions}

The results of this study suggest that a single pulse dose of clomipramine significantly can ameliorate the symptoms of depression within one week in inpatients with moderate to severe depression with only mild side effects. This pulse regime may therefore be an alternative to ECT. Limitations of the study were the small number of patients and 


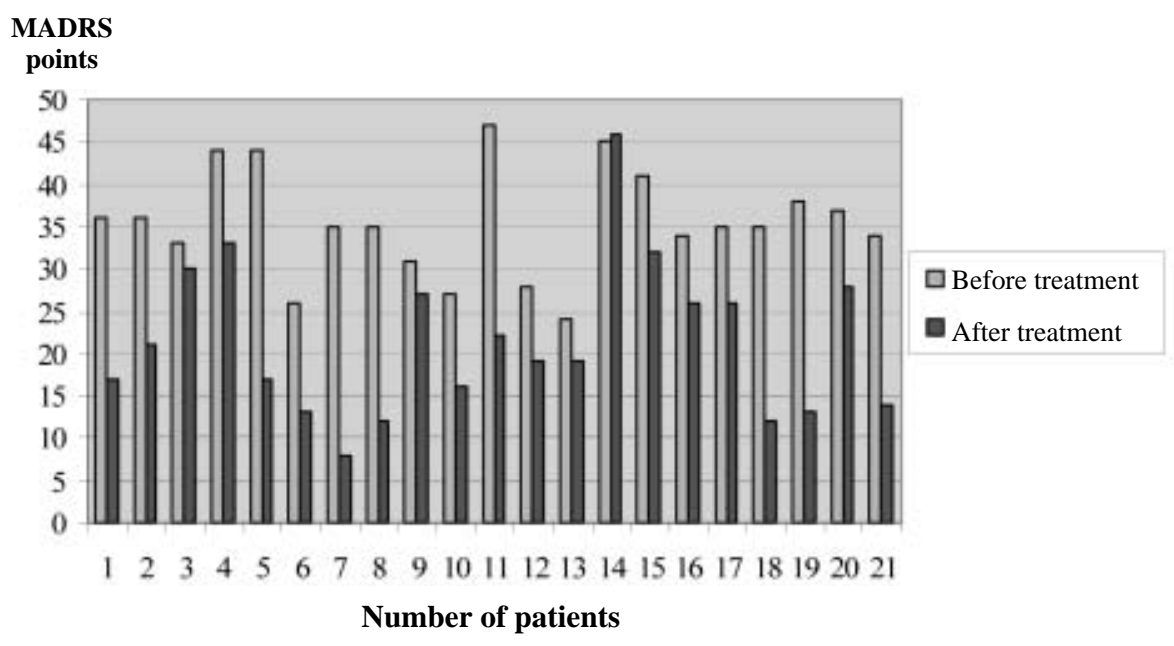

Figure 1. MADRS before and after treatment.

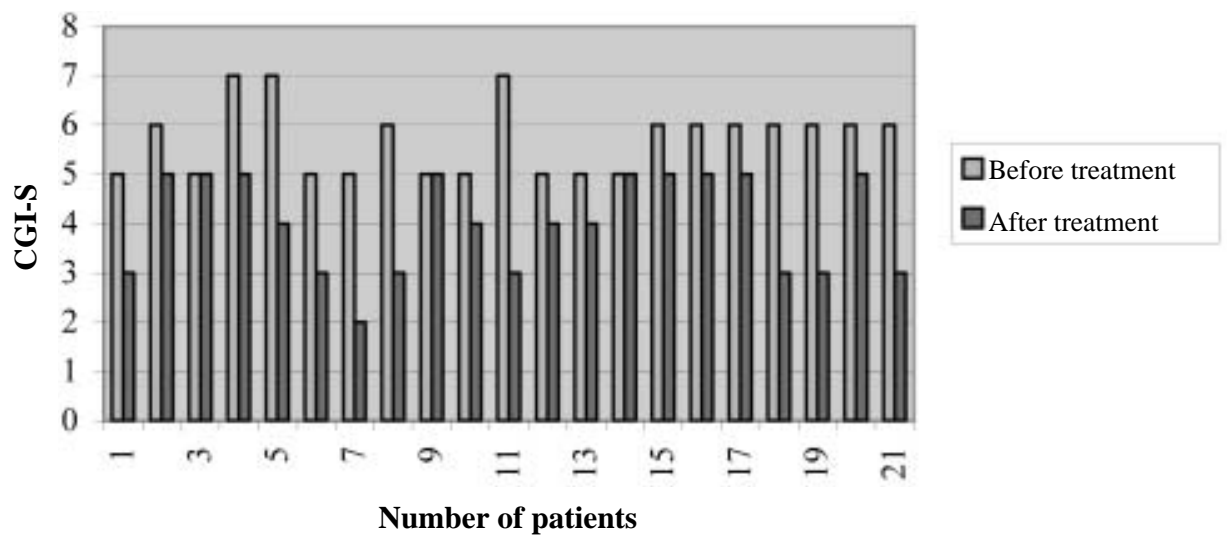

Figure 2. CGI-S before and after treatment.

the lack of a placebo control group. Further studies are warranted.

\section{References}

1. Blier P, de Montigny C, Chaput Y. Modifications of the serotonin system by antidepressant treatment: Implications for the therapeutic response in major depression. J Clin Psychopharmacol 1987; 7: 24S-35S.
2. Chiodo LA, Antelman SM. Repeated tricyclics induce a progressive dopamine autoreceptorsubsensitivity independent of daily drug treatment. Nature 1980; 287(5781): 451-4.

3. Koran L, Pallanti S, Paiva RS, Quercioli L. Pulse loading versus gradual dosing of intravenous clomipramine in obsessive-compulsive disorder. Eur Neuropsychopharmacol 1998; 8(2): 121-6.

4. Eriksson E. Strong but non-selecitve serotonin reuptake inhibition: the unique profile of clomipramine. Anafranil (clomipramine); Past, present and future, a product profile. Basel: Ciba-Geigy 1994; 86-93. 
5. Dencker SJ, Nagy A. Single versus divided daily dosages of clomipramine. Plasma concentration and clinical effect. Acta Psychiatr Scand 1979; 59(3): 326-34.

6. Pollock B, Perel J, Nathan S, Kupfer D. Acute antidepressant effect following pulse loading with intravenous and oral clomipramine. Arch Gen Psychiatry 1989; 46(1): 29-35.

7. Hamilton M. Development of a rating scale for primary depressive illness. Br J Soc Clin Psychol 1967; 6(4): 278-296.

8. Sallee FR, Vridavanam N, Deas-Nesmith D, Carson $\mathrm{S}$, Sethuraman G. Pulse intravenous clomipramine for depressed adolescents: Double-blind, controlled trial. Am J Psychiatry 1997;154 (5): 668-673.
9. Montgomery S, Asberg M. A new depression scale designed to be sensitive to change. Br J Psychiatr 1979; 134: s382-9.

10. Guy, W. Clinical Global Impressions. In ECDEU Assessment Manual for Psychopharmacology, revised. Rockville, MD. National Institute of Mental Health. 1976.

Address for correspondence:

Maj-Liz Persson

Karolinska Institutet, the Department of Clinical Neuroscience, Division of Psychiatry, Karolinska University Hospital in Huddinge, S-141 86 Stockholm, Sweden.

Tel: +46 70 4843213, fax: +46855093019

E-mail: maj-liz.persson@sll.se 